\title{
Article \\ A New Method to Verify the Measurement Speed and Accuracy of Frequency Modulated Interferometers
}

\author{
Toan-Thang Vu ${ }^{1}$, Thanh-Tung Vu ${ }^{1, *(\mathbb{D}}$, Van-Doanh Tran ${ }^{1}$, Thanh-Dong Nguyen ${ }^{1}$ and Ngoc-Tam Bui ${ }^{1,2, *(1)}$ \\ 1 School of Mechanical Engineering, Hanoi University of Science and Technology, Hanoi 100000, Vietnam; \\ thang.vutoan@hust.edu.vn (T.-T.V.); doanh.tvcb170290@sis.hust.edu.vn (V.-D.T.); \\ dong.nguyenthanh@hust.edu.vn (T.-D.N.) \\ 2 Shibaura Institute of Technology, College of Systems Engineering and Science, Tokyo 135-8548, Japan \\ * Correspondence: tung.vuthanh@hust.edu.vn (T.-T.V.); tambn@shibaura-it.ac.jp (N.-T.B.)
}

Citation: Vu, T.-T.; Vu, T.-T.; Tran, V.-D.; Nguyen, T.-D.; Bui, N.-T. A New Method to Verify the Measurement Speed and Accuracy of Frequency Modulated Interferometers. Appl. Sci. 2021, 11 5787. https://doi.org/10.3390/ app11135787

Academic Editor: Jacek Wojtas

Received: 3 June 2021

Accepted: 19 June 2021

Published: 22 June 2021

Publisher's Note: MDPI stays neutral with regard to jurisdictional claims in published maps and institutional affiliations.

Copyright: (c) 2021 by the authors. Licensee MDPI, Basel, Switzerland. This article is an open access article distributed under the terms and conditions of the Creative Commons Attribution (CC BY) license (https:// creativecommons.org/licenses/by/ $4.0 /)$.

\begin{abstract}
The measurement speed and measurement accuracy of a displacement measuring interferometer are key parameters. To verify these parameters, a fast and high-accuracy motion is required. However, the displacement induced by a mechanical actuator generates disadvantageous features, such as slow motion, hysteresis, distortion, and vibration. This paper proposes a new method for a nonmechanical high-speed motion using an electro-optic modulator (EOM). The method is based on the principle that all displacement measuring interferometers measure the phase change to calculate the displacement. This means that the EOM can be used to accurately generate phase change rather than a mechanical actuator. The proposed method is then validated by placing the EOM into an arm of a frequency modulation interferometer. By using two lock-in amplifiers, the phase change in an EOM and, hence, the corresponding virtual displacement could be measured by the interferometer. The measurement showed that the system could achieve a displacement at $20 \mathrm{kHz}$, a speed of $6.08 \mathrm{~mm} / \mathrm{s}$, and a displacement noise level $<100 \mathrm{pm} / / \sqrt{ } \mathrm{Hz}$ above $2 \mathrm{kHz}$. The proposed virtual displacement can be applied to determine both the measurement speed and accuracy of displacement measuring interferometers, such as homodyne interferometers, heterodyne interferometers, and frequency modulated interferometers.
\end{abstract}

Keywords: electro-optic modulator; frequency modulation; displacement measuring interferometer

\section{Introduction}

High-precision technology is increasingly critical for industrial applications. The demand for high-speed and precise processing has been on the rise in various fields. To meet these requirements, different types of displacement measuring sensors are available, including capacitive sensors, linear encoders, and laser interferometers. In a short measurement range, capacitive sensors can obtain sub-nanometer resolution [1,2]. There are some disadvantages of capacitive sensors, such as sensitivity to temperature and humidity, short stand-off distance, and relatively low bandwidth [3,4]. Linear encoders, which can measure both distance and displacement at nanometer resolution over a long measurement range, are widely used as machine tools $[5,6]$. However, the complex structure and large volume limits their application in ultra-precision measurements. Among these sensors, displacement measuring interferometers have increasingly been adopted because of their high level of accuracy and traceability to the definition of the meter. Numerous displacement measuring interferometers have been developed, such as homodynes [7-9], heterodynes [10-12], and frequency/phase modulation interferometers [13-16]. In open-air environments, the measurement range and accuracy of the homodyne interferometer are reduced due to the refractive index fluctuation [17]. The heterodyne frequency is less than $20 \mathrm{MHz}$, and, hence, the measurement speed is approximately $5 \mathrm{~m} / \mathrm{s}[18,19]$. For a frequency modulation interferometer, the measurement speed is limited by the modulation frequency of the laser source. Laser diodes (LDs) are widely used as the laser source in interferometers due 
to their advantageous features, such as high power, compactness, and long lifespan,. In particular, the high modulation frequency of LDs can be obtained by applying a current modulation at the $\mathrm{GHz}$ level $[20,21]$. Hence, the measurement speed of the frequency modulation interferometer can theoretically reach a level of $10 \mathrm{~m} / \mathrm{s}$. Therefore, a considerable challenge for the interferometer is the measurement speed verification at nanoscale uncertainty. Normally, a piezo-electric (PZT) actuator uses the motion of the mirrors for interferometry because of its high motion resolution. However, the travelling speed of the PZT stage is limited to several $\mathrm{kHz}$. In addition, a mechanical displacement PZT system typically shows hysteresis, which is a type of cyclic error [22-24]. Hysteresis introduces uncertainties into measurements, which should be suppressed or removed. Voice coil actuators are also used for many high-precision motion applications [25,26]. The actuator can achieve a resolution of $2 \mathrm{~nm}$ over a range of $1 \mathrm{~mm}$ [27]. Two major disadvantages of voice coil actuators are heat output and disturbance from moving wires [28]. Hence, voice coil actuators are not suitable for wide-range nano-positioning at high speed.

The measurement speed and measurement precision of displacement measuring interferometers are key parameters. For interferometers, the measurement speed or rate implies the time required to take one displacement measurement in the unit of seconds or $\mathrm{Hz}$. In our previous works, a high modulation frequency of $3 \mathrm{MHz}$ was successfully applied to the LD to improve the measurement speed of the frequency modulated interferometer $[13,14,29]$. Moreover, a high-precision phase meter was developed to measure the phase change in the interferometer [12]. To verify the measurement accuracy of our proposed interferometers, the displacement of the target mirror was measured using a capacitive sensor (D100, Physik Instrumente) integrated into the PZT stage. However, the bandwidth of the capacitive sensor was less than $3 \mathrm{kHz}$, and the resolution was $1 \mathrm{~nm}$ over a displacement range of $2 \mu \mathrm{m}[13,14]$. Therefore, it remains a significant challenge to verify both parameters using a nanometer resolution and high-speed displacement actuator. In this paper, a high-speed virtual displacement without hysteresis is proposed and validated. It is noteworthy that all types of displacement interferometers determine target displacement by measuring the phase shift. A pure phase modulation at high frequency can be generated using an electro-optic modulator (EOM). This means that the EOM can be used to induce the phase shift rather than mechanical motions, such as PZT or voice coil actuators, to confirm the measurement speed and accuracy of displacement measuring interferometers. By using an EOM, the phase shift can be generated ranging from several $\mathrm{kHz}$ to some tens of $\mathrm{MHz}$ without distortion, hysteretic, or vibration. Hence, both the measurement accuracy and speed of the interferometer under assessment can be accurately verified. In the primary experiment, an EOM was placed into an arm of a frequency modulated interferometer. The virtual displacement was implemented using the EOM, in which the phase was changed using a modulation voltage at $20 \mathrm{kHz}$. Using two lock-in amplifiers (LIAs) and a Lissajous diagram, the phase change in the EOM and the corresponding virtual displacement could be measured using the frequency modulated interferometer.

In summary, the main contributions of this paper are listed as follows: (1) the calculation and design of a virtual displacement generated using an EOM; (2) the validation and measurement of the virtual displacement using a frequency modulated interferometer; (3) the comparison of the resulting measurement using the interferometer and theoretical result of the motion. Both the measurement speed and accuracy of the frequency modulated interferometer can be verified using the proposed method. This is significant in high-speed measuring applications, such as spindle error measurement, vibration measurement, and machine tool calibration.

\section{Methodology}

Figure 1a shows the schematic of the frequency modulated interferometer. The laser source was a laser diode (LD), in which the frequency was modulated with a sinusoidal signal by a modulation current injection. The angle frequency $\omega(t)$ of the LD is expressed as

$$
\omega(t)=\omega_{0}+\Delta \omega \sin (\omega t)
$$


where $\omega_{0}\left(=2 \pi f_{0}\right), \omega$, and $\Delta \omega$ are the initial angle frequency of the $L D$, acting as the carrier angle frequency, the angle modulation frequency, and the angle modulation frequency excursion of $\omega$, respectively. An isolator was used to prevent unwanted lights returning to the LD. A half-wave plate (HWP) was employed to rotate the polarization plane of the incident light at $45^{\circ}$. A beam splitter (BS) divided the incident light from the isolator into two beams: a reflected beam and a transmitted beam. The reflected beam was sent to a mirror (M1) in the reference arm. In the measurement arm, the second beam was sent to another mirror (M2) along a polarizer (P) and an EOM. The polarizer was placed in front of the EOM to ensure the correct polarization of the input light. After reflection from the mirrors, the reflected lights of the two arms recombined with each other at the BS to generate interference.

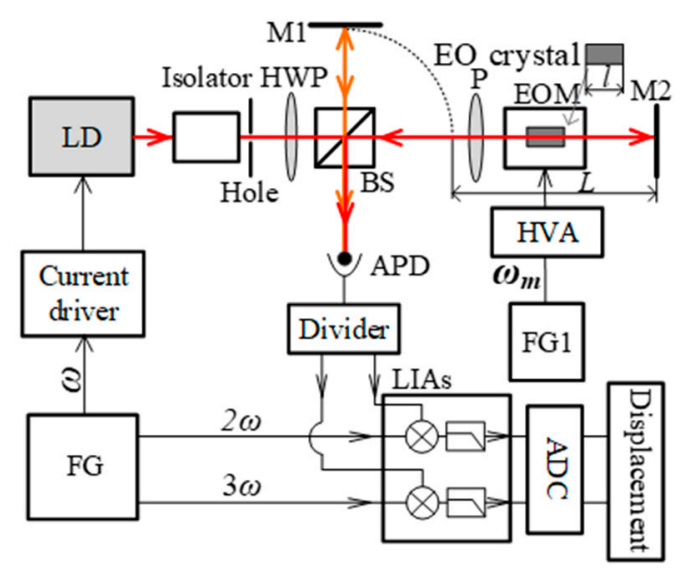

(a)

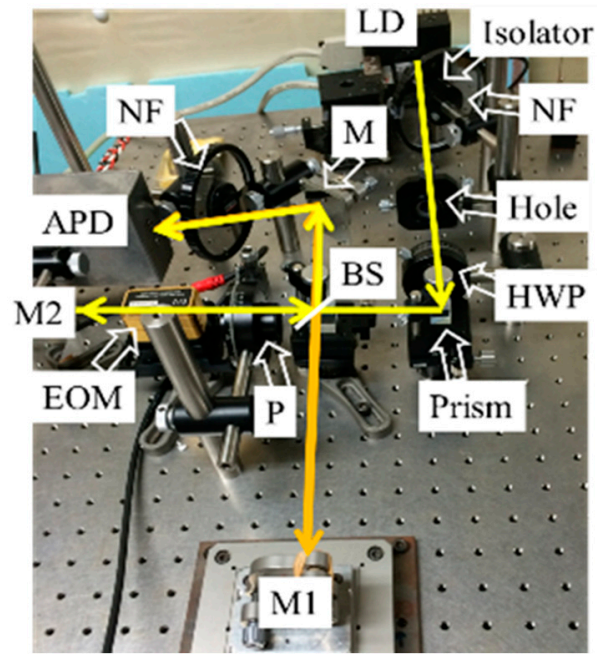

(b)

Figure 1. Virtual displacement-measuring interferometer using a frequency-modulated laser source. (a) Schematic design and (b) the experimental setup. LD: laser diode; HWP: half-wave plate; BS: beam splitter; M: mirror; P: polarizer; EOM: electro-optic modulator; EO crystal: electro-optic crystal; NF: neutral density filter; HVA: high-voltage amplifier; FG: function generator; APD: avalanche photodetector; LIA: lock-in amplifier; ADC: analog-to-digital converter.

The intensity $I(\tau, t)$ of the interference signal $[13,14]$ is written as

$$
\begin{aligned}
& I(\tau, t)=E^{2} 01+E^{2} 02+2 E_{01} E_{02} \cos \left(\omega_{0} \tau\right) J_{0}(m)+4 E_{01} E_{02} \cos \left(\omega_{0} \tau\right) \sum_{n=1}^{\infty} J_{2 n}(m) \cos (2 n \omega t) \\
& -4 E_{01} E_{02} \sin \left(\omega_{0} \tau\right) \sum_{n=1}^{\infty} J_{2 n-1}(m) \cos [(2 n-1) \omega t],
\end{aligned}
$$

where $\tau, m, E_{01}$ and $E_{02}, n, J_{0}(m), J_{2 n}(m)$, and $J_{2 n-1}(m)$ are the changes in time between the two arms of the interferometer, the modulation index, the amplitudes of the electric fields in the reference and measurement arms, an integer, and the Bessel functions, respectively. Here,

$$
m=\frac{\Delta \omega}{\omega} \sin \left(\frac{\omega \tau}{2}\right) \approx \frac{2 \pi \Delta f n_{\text {air }} L}{c}
$$

where $\Delta f(\Delta \omega=2 \pi \Delta f), n_{\text {air }}, L$, and $c$ are the frequency modulation excursion, the refractive index of air, the unbalance length of the interferometer, and the speed of light in a vacuum. A divider split $I(\tau, t)$ into two parts, which are coupled with the two purely sinusoidal signals of $2 \omega$ and $3 \omega$ from the function generator. By using the two LIAs [13,14], the intensities $I_{2} \omega$ and $I_{3 \omega}$ of the $2 \omega$ and $3 \omega$ harmonics of $I(\tau, t)$, respectively, are produced as follows:

$$
\begin{gathered}
I_{2 \omega}=2 E_{01} E_{02} \cos \left(\omega_{0} \tau\right) J_{2}(m), \\
I_{3 \omega}=-2 E_{01} E_{02} \sin \left(\omega_{0} \tau\right) J_{3}(m) .
\end{gathered}
$$


Here, $J_{2}(m)$ and $J_{3}(m)$ are the second- and third-order Bessel functions, respectively. By using the Lissajous diagram, $\omega_{0} \tau$ is determined. The total phase difference $\Phi$ between the arms is

$$
\Phi=\omega_{0} \tau=\arctan \left(-\frac{I_{3 \omega}}{I_{2 \omega}} \cdot \frac{J_{2}(m)}{J_{3}(m)}\right) .
$$

To generate the virtual displacement, the EOM was applied using a sinusoidal voltage $V(t)$

$$
V(t)=V_{E O M} \sin \left(\omega_{E O M} t\right),
$$

where $V_{E O M}$ is the amplitude of $V(t)$. For the EOM (4002, Newport) [30], the optical phase difference $\Delta \Phi_{1}$ induced by applying $V(t)$ is

$$
\Delta \Phi_{1}=\frac{\pi}{V_{\pi}} V,
$$

where $V_{\pi}$ is a half-wave voltage. Because the laser beam in the measurement arm was double-phase modulated (Figure 1), by substituting Equation (6) into Equation (7), the phase $\Delta \Phi_{E O M}=2 \Delta \Phi_{1}$ is

$$
\Delta \Phi_{E O M}=2 \Delta \Phi_{1}=\frac{2 \pi}{V_{\pi}} V=\frac{2 \pi}{V_{\pi}} V_{E O M} \sin \left(\omega_{E O M} t\right) .
$$

However, the total phase difference $\Phi$ between the arms of the interferometer is

$$
\Phi=\omega_{0} \tau=\frac{4 \pi n_{\text {air }}(L-l)}{\lambda}+\frac{4 \pi}{\lambda}\left(n_{e}+\Delta n_{e}\right) l=\frac{4 \pi n_{\text {air }}(L-l)}{\lambda}+\frac{4 \pi}{\lambda} n_{e} l+\Delta \Phi_{E O M},
$$

where $L, n_{\text {air }}, l, n_{e}, \Delta n_{e}$, and $\lambda$ are the unbalance length of the interferometer, the refractive index of air, the length and unperturbed refractive index of the electro-optic (EO) crystal, the change in $n_{e}$ induced by applying $V(t)$, and the laser wavelength, respectively. Here,

$$
\Delta \Phi_{E O M}=\frac{4 \pi}{\lambda} \Delta n_{e} l=\frac{4 \pi}{\lambda} \Delta l
$$

$\Delta l$ is the change in the optical path in the EO crystal, which is defined as the virtual displacement of M2 due to applying $V(t)$. Substituting Equation (8) into Equation (10), $\Delta l$ becomes

$$
\Delta l=\frac{\lambda}{2} \frac{V_{0}}{V_{\pi}} \sin \left(\omega_{E O M} t\right) .
$$

Substituting Equations (5) and (10) into Equation (9), $\Delta l$ measured by the interferometer is

$$
\Delta l=\frac{\lambda}{4 \pi}\left\{\arctan \left(-\frac{I_{3 \omega}}{I_{2 \omega}} \cdot \frac{J_{2}(m)}{J_{3}(m)}\right)\right\}-\left[(L-l) n_{\text {air }}+n_{e} l\right] .
$$

In this experiment, we compared the measurement in Equation (12) with the calculated displacement in Equation (11) at the high phase-modulation frequency $\omega_{m}$.

For the frequency modulated interferometer, the maximum measurable speed $V_{\max }$ can be given by [13]

$$
V_{\max } \leq k \frac{\lambda}{4} f,
$$

where $k$ and $f$ are an integer and the modulation frequency of LD, respectively. Here, $k$ represents the ratio of the cutoff frequency of the LIAs and the modulation frequency of LD; normally $k=0.1-0.8$. In this paper, a virtual displacement was developed at a speed of tens of $\mathrm{kHz}$ and without hysteresis generated using an EOM.

\section{Experiments and Results}

A photograph of the experimental setup is shown in Figure 1b. The laser source was an LD (HL6312G, Thorlabs Inc., Newton, NJ, USA), and it was frequency modulated with a sine-wave signal (a frequency modulation of $20 \mathrm{MHz}$ and modulation excursion of $570 \mathrm{MHz}$ ) by modulating the injection current. To produce a high-speed virtual motion, the $4002 \mathrm{EOM}$ with $\mathrm{V}_{\pi}=\sim 125 \mathrm{~V}$ at $\lambda=635 \mathrm{~nm}$ was used. The EOM was phase changed using a $20 \mathrm{kHz}$ sine-wave signal $\mathrm{V}(\mathrm{t})$, which had $\mathrm{V}_{0}=2 \mathrm{~V}$, by utilizing a digital function generator (Moku:labs, Liquid Instruments), and it was then amplified 30 times by a high-voltage amplifier. $\Delta \Phi_{\mathrm{EOM}}$ was produced, and the $20 \mathrm{kHz}$ sine-wave virtual displacement $\Delta \mathrm{l}$ could thus be attained with a peak-to-peak ( $\mathrm{p}-\mathrm{p}$ ) amplitude of $\sim 152 \mathrm{~nm}(=635 \mathrm{~nm} \times 2 \mathrm{~V} \times 30 / 2 / 125 \mathrm{~V})$. The $\mathrm{I}(\tau, \mathrm{t})$ was detected using an avalanche photodetector (DET08CFC/M, Thorlabs). To extract $\mathrm{I}_{2} \omega(2 \omega=40 \mathrm{MHz})$ and $\mathrm{I}_{3 \omega}(2 \omega=60 \mathrm{MHz})$, we used two digital LIAs (Moku:labs, Liquid Instruments), which were synchronized with each other and had a 
cutoff frequency of $200 \mathrm{kHz}$. The $\mathrm{I}_{2} \omega$ and $\mathrm{I}_{3 \omega}$ were then recorded using an analog-to-digital converter (AD16-16U(PCI)EV, Contec Co., Osaka, JP) at a sampling frequency of $715 \mathrm{kHz}$ to eliminate MHzorder noises in the harmonics. In this experiment, the modulation index was $\mathrm{m} \approx 2.4$. This led to $\mathrm{J}(2 \omega)=2.15 \times \mathrm{J}(3 \omega)$. In addition, because $(\mathrm{L}-\mathrm{l}) \mathrm{n}_{\text {air }}+\mathrm{n}_{\mathrm{e}} \mathrm{l} \approx \mathrm{n}_{\text {air }} \mathrm{L} \approx 0.2 \mathrm{~m}$. Equation (13) is rewritten as

$$
\Delta l=\frac{\lambda}{4 \pi}\left\{\arctan \left(-\frac{1}{2.15} \frac{I_{3 \omega}}{I_{2 \omega}}\right)\right\}-L n_{\text {air }}
$$

The experiments were performed under the conditions in Table 1. The second and third harmonics that were clearly detected by LIAs are shown in Figure 2a. A Lissajous diagram using the two harmonics is shown in Figure $2 \mathrm{~b}$. A sine-wave displacement at $20 \mathrm{kHz}$ and a p-p amplitude of $\sim 152 \mathrm{~nm}$ was obtained over $1 \mathrm{~ms}$, as shown in Figure 3a. This led to a measured speed of $6.08 \mathrm{~mm} / \mathrm{s}$ $\left(=2 \Delta l \times f_{E O M}=2 \times 152 \mathrm{~nm} \times 20 \mathrm{kHz}\right)$.

Table 1. The experimental conditions for the virtual displacement measurement.

\begin{tabular}{ll} 
Modulation frequency $\omega$ for LD & $2 \pi \times 20 \mathrm{MHz}$ \\
Frequency modulation excursion $\Delta \omega$ & $2 \pi \times 570 \mathrm{MHz}$ \\
Cutoff frequency of LIAs & $200 \mathrm{kHz}$ \\
$V_{\pi}$ at $536 \mathrm{~nm}$ & $125 \mathrm{~V}$ \\
Applied angle frequency for $\omega_{E O M}$ & $2 \pi \times 20 \mathrm{kHz}$ \\
Unbalanced length $L$ & $0.2 \mathrm{~m}$ \\
Working sampling frequency & $715 \mathrm{kHz}$ \\
\hline
\end{tabular}

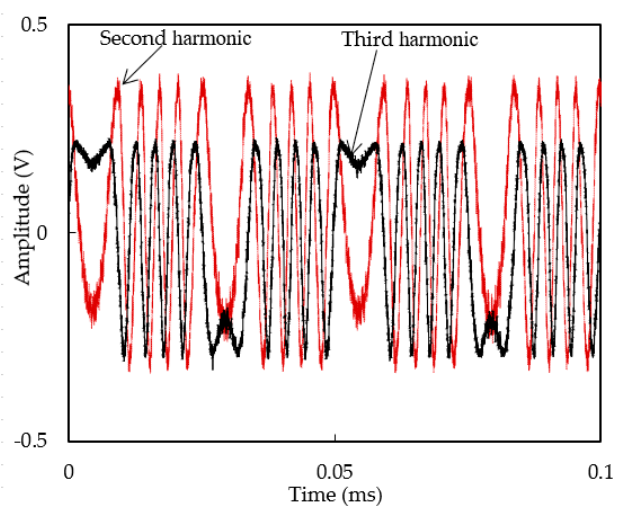

(a)

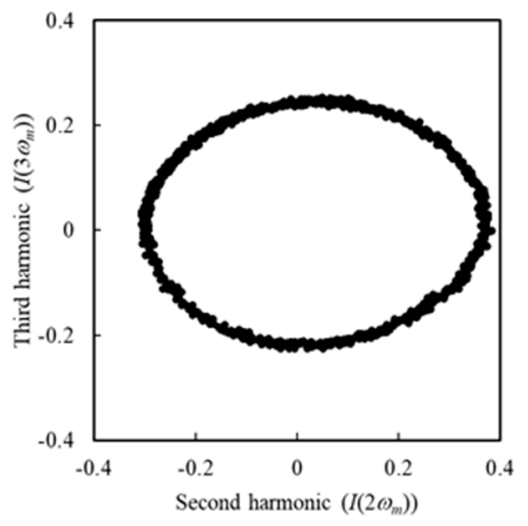

(b)

Figure 2. Harmonic detection: (a) second and third harmonics; (b) Lissajous diagram using $I_{2} \omega$ and $I_{3} \omega$ harmonics.

The result obtained by the interferometer was compared with a calculated displacement function $F$ using Equation (11), $F=A+B \times \sin (2 \pi \times 20 \mathrm{kHz} \times t)$, where $A=-0.152 \mu \mathrm{m}$ and $B=-0.076 \mu \mathrm{m}$. The difference between the measured result and $F$ is shown in Figure $3 \mathrm{~b}$. A displacement difference of $\sim 30 \mathrm{~nm}$ and a standard deviation of $\sim 6 \mathrm{~nm}$ were attained. The displacement noise floor analyzed in Figure $3 b$ is depicted in Figure $3 c$. Above $2 \mathrm{kHz}$, a displacement noise floor of less than $100 \mathrm{pm} / \sqrt{ } \mathrm{Hz}$ was achieved. A dominated noise peak at $20 \mathrm{kHz}$ can be seen. This led to the cycle error of the sine-wave displacement in Figure $3 \mathrm{a}$, and this noise level was $\sim 100 \mathrm{pm} / \sqrt{ } \mathrm{Hz}$. The result showed that our method could generate a stable and high-precision displacement that could be used as a reference. Even though unwanted noises caused by vibration and air disturbance still existed in the measurement, the method was promising for high-speed measurements. 


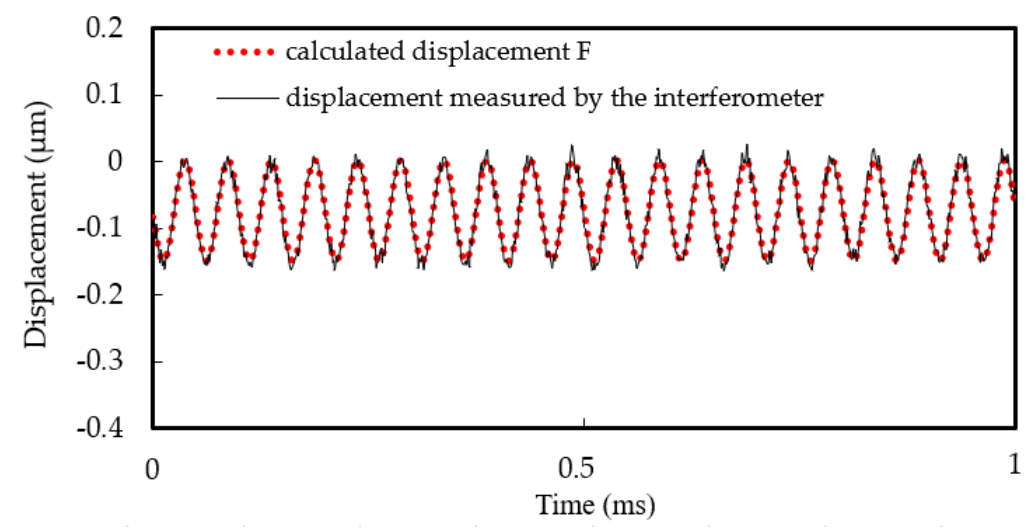

(a)

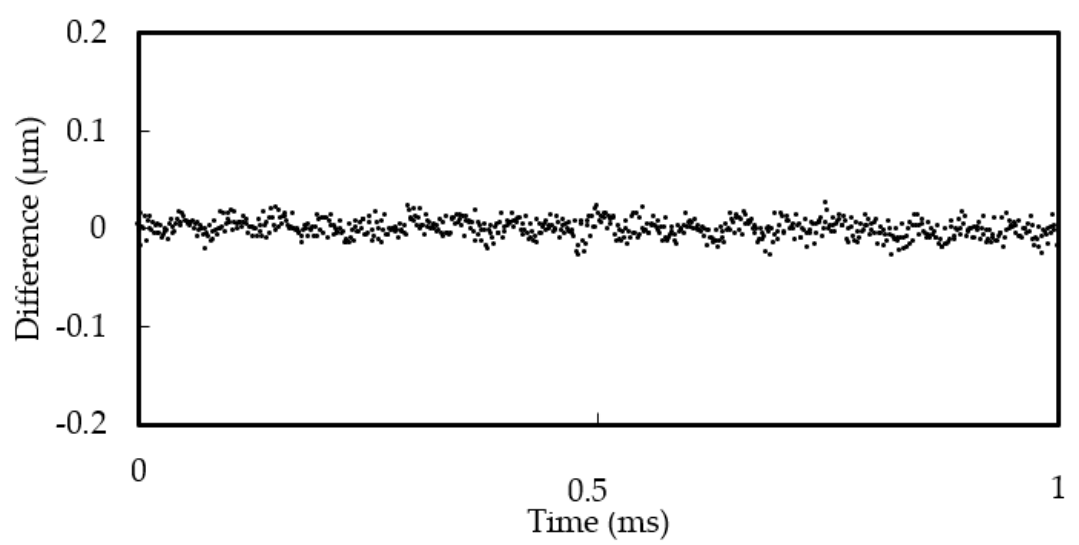

(b)

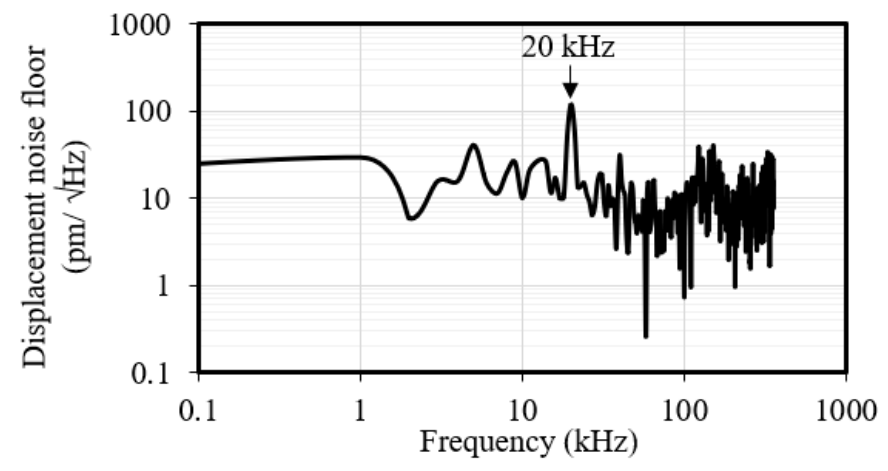

(c)

Figure 3. Measurement: (a) virtual displacement measured by the interferometer (solid line) compared with the calculated displacement function $F=A+B \times \sin (2 \pi \times 20 \mathrm{kHz} \times t)$ (dot line; see Equation (11), where $A=-0.152 \mu \mathrm{m}$ and $B=-0.076 \mu \mathrm{m}) ;(\mathbf{b})$ difference between the result obtained by the interferometer and F; and (c) displacement noise floor analyzed from (b) by Fourier transform.

From Equation (5), when $k=0.8, \lambda=635 \mathrm{~nm}$, and $f=20 \mathrm{MHz}$, the maximum measurable speed of $2.54 \mathrm{~m} / \mathrm{s}$ can be achieved. However, due to the limitation of the hardware caused by the low bandwidth of the high-voltage amplifier (HVA) and LIAs, we could not perform a virtual displacement of several $\mathrm{m} / \mathrm{s}$. We plan to use a higher bandwidth HVA and LIAs to improve the measurement speed. Another disadvantage of the proposed method is that the use of the EOM in one arm of the interferometer can cause the polarization mixing effect, and, hence, it may induce some noise of the interference signal. To improve the interference signal, some polarization optics can be used in the next experiment. 


\section{Conclusions}

In this study, high-speed displacement generation using an electro-optic modulator (EOM) was proposed and validated. A frequency modulated interferometer was established to measure the virtual displacement produced by the EOM. To produce a high-speed displacement, an EOM was phase changed using a high-speed sinusoidal modulation voltage. A $20 \mathrm{kHz}$ sine-wave signal with an amplified amplitude of $60 \mathrm{~V}$ was applied to the EOM. A corresponding sinusoidal virtual displacement of $\sim 152 \mathrm{~nm}$ at $20 \mathrm{kHz}$ was then generated. The interferometer extracted an interference signal that contained the phase change in the EOM. Two LIAs and a Lissajous diagram were adopted for detecting the phase change and calculating the virtual displacement. The measurement showed that the virtual displacement, with an amplitude of $152 \mathrm{~nm}$ at $20 \mathrm{kHz}$ and a measurement speed of $6.08 \mathrm{~mm} / \mathrm{s}$, was successfully measured by the interferometer. A displacement noise floor < $100 \mathrm{pm} / \sqrt{ } \mathrm{Hz}$ above $2 \mathrm{kHz}$ was achieved. This experiment was successful at developing a displacement reference at high speed. For future study, the possibility of using a higher modulation for the LDs and the residual amplitude modulation effect will be investigated, as well as high-speed mechanical displacements when the relevant hardware is available.

Author Contributions: Conceptualization, T.-D.N. and T.-T.V. (Thanh-Tung Vu ); methodology, T.T.V. (Toan-Thang Vu); software, V.-D.T.; validation, T.-D.N, and V.-D.T.; formal analysis, N.-T.B.; investigation, T.-T.V. (Thanh-Tung Vu ); resources, N.-T.B.; data curation, V.-D.T.; writing-original draft preparation, T.-T.V. (Toan-Thang Vu); writing-review and editing, T.-T.V. (Thanh-Tung Vu ); visualization, T.-T.V. (Toan-Thang Vu); funding acquisition, N.-T.B. All authors have read and agreed to the published version of the manuscript.

Funding: This research was funded by Hanoi University of Science and Technology, grant number T2020-PC-201.

Institutional Review Board Statement: Not applicable.

Informed Consent Statement: Not applicable.

Data Availability Statement: This study did not report any data.

Acknowledgments: This work was supported by the Centennial SIT Action for the 100th anniversary of Shibaura Institute of Technology to enter the top ten Asian Institute of Technology.

Conflicts of Interest: The authors declare no conflict of interest.

\section{References}

1. Peng, K.; Yu, Z.; Liu, X.; Chen, Z.; Pu, H. Features of capacitive displacement sensing that provide high-accuracy measurements with reduced manufacturing precision. IEEE Trans. Ind. Electron. 2017, 64, 7377-7386. [CrossRef]

2. Ye, Y.; Zhang, C.; He, C.; Wang, X.; Huang, J.; Deng, J. A review on applications of capacitive displacement sensing for capacitive proximity sensor. IEEE Access 2020, 8, 45325-45342. [CrossRef]

3. Chi, C.; Sun, X.; Xue, N.; Li, T.; Liu, C. Recent progress in technologies for tactile sensors. Sensors 2018, 18, 948. [CrossRef]

4. Zhang, D.; Zhao, S.; Zheng, Q.; Lin, L. Absolute capacitive grating displacement measuring system with both high-precision and long-range. Sens. Actuators A Phys. 2019, 295, 11-22. [CrossRef]

5. Ye, G.; Liu, H.; Ban, Y.; Shi, Y.; Yin, L.; Lu, B. Development of a reflective optical encoder with submicron accuracy. Opt. Commun. 2018, 411, 126-132. [CrossRef]

6. Khouygani, M.H.G.; Jeng, J.Y. High-precision miniaturized low-cost reflective grating laser encoder with nanometric accuracy. Appl. Opt. 2020, 59, 5764-5771. [CrossRef]

7. Hori, Y.; Gonda, S.; Bitou, Y.; Watanabe, A.; Nakamura, K. Periodic error evaluation system for linear encoders using a homodyne laser interferometer with 10 picometer uncertainty. Precis. Eng. 2018, 51, 388-392. [CrossRef]

8. Yan, L.; Chen, B.; Chen, Z.; Xie, J.; Zhang, E.; Zhang, S. Phase-modulated dual-homodyne interferometer without periodic nonlinearity. Meas. Sci. Technol. 2017, 28, 115006. [CrossRef]

9. Lou, Y.; Yan, L.; Chen, B. A phase modulating homodyne interferometer with tilting error compensation by use of an integrated four-photodetector. Rev. Sci. Instrum. 2019, 90, 025111. [CrossRef]

10. Joo, K.N.; Clark, E.; Zhang, Y.; Ellis, J.D.; Guzmán, F. A compact high-precision periodic-error-free heterodyne interferometer. JOSA A 2020, 37, B11-B18. [CrossRef]

11. Yokoyama, S.; Hori, Y.; Yokoyama, T.; Hirai, A. A heterodyne interferometer constructed in an integrated optics and its metrological evaluation of a picometre-order periodic error. Precis. Eng. 2018, 54, 206-211. [CrossRef] 
12. Nguyen, T.D.; Duong, Q.A.; Higuchi, M.; Vu, T.T.; Wei, D.; Aketagawa, M. 19-picometer mechanical step displacement measurement using heterodyne interferometer with phase-locked loop and piezoelectric driving flexure-stage. Sens. Actuators A Phys. 2020, 304, 111880. [CrossRef]

13. Vu, T.T.; Higuchi, M.; Aketagawa, M. Accurate displacement-measuring interferometer with wide range using an I2 frequencystabilized laser diode based on sinusoidal frequency modulation. Meas. Sci. Technol. 2016, 27, 105201. [CrossRef]

14. Duong, Q.A.; Vu, T.T.; Higuchi, M.; Wei, D.; Aketagawa, M. Iodine-frequency-stabilized laser diode and displacement-measuring interferometer based on sinusoidal phase modulation. Meas. Sci. Technol. 2018, 29, 065204. [CrossRef]

15. Zhang, S.; Yan, L.; Chen, B.; Xu, Z.; Xie, J. Real-time phase delay compensation of PGC demodulation in sinusoidal phasemodulation interferometer for nanometer displacement measurement. Opt. Express 2017, 25, 472-485. [CrossRef]

16. Xu, J.; Huang, L.; Yin, S.; Gao, B.; Chen, P. All-fiber self-mixing interferometer for displacement measurement based on the quadrature demodulation technique. Opt. Rev. 2018, 25, 40-45. [CrossRef]

17. Jang, Y.S.; Kim, S.W. Compensation of the refractive index of air in laser interferometer for distance measurement, A review. Int. J. Precis. Eng. Manuf. 2017, 18, 1881-1890. [CrossRef]

18. Demarest, F.C. High-resolution, high-speed, low data age uncertainty, heterodyne displacement measuring interferometer electronics. Meas. Sci. Technol. 1998, 9, 1024. [CrossRef]

19. Topcu, S.; Chassagne, L.; Haddad, D.; Alayli, Y.; Juncar, P. Heterodyne interferometric technique for displacement control at the nanometric scale. Rev. Sci. Instrum. 2003, 74, 4876-4880. [CrossRef]

20. Chan, S.C.; Liu, J.M. Frequency modulation on single sideband using controlled dynamics of an optically injected semiconductor laser. IEEE J. Quantum Electron. 2006, 42, 699-705. [CrossRef]

21. D'Amato, F.; De Rosa, M. Tunable diode lasers and two-tone frequency modulation spectroscopy applied to atmospheric gas analysis. Opt. Lasers Eng. 2002, 37, 533-551. [CrossRef]

22. Yan, P.; Zhang, Y. High precision tracking of a piezoelectric nano-manipulator with parameterized hysteresis compensation. Smart Mater. Struct. 2018, 27, 065018. [CrossRef]

23. Cai, K.; Tian, Y.; Wang, F.; Zhang, D.; Liu, X.; Shirinzadeh, B. Modeling and tracking control of a novel XY $\theta z$ stage. Microsyst. Technol. 2017, 23, 3575-3588. [CrossRef]

24. Lin, C.; Yu, J.; Wu, Z.; Shen, Z. Decoupling and control of micromotion stage based on hysteresis of piezoelectric actuation. Microsyst. Technol. 2019, 25, 3299-3309. [CrossRef]

25. Teo, T.J.; Chen, I.M.; Yang, G.; Lin, W. A flexure-based electromagnetic linear actuator. Nanotechnology 2008, 19, 315501. [CrossRef]

26. Youm, W.; Jung, J.; Lee, S.; Park, K. Control of voice coil motor nanoscanners for an atomic force microscopy system using a loop shaping technique. Rev. Sci. Instrum. 2008, 79, 13706-13707. [CrossRef]

27. Fukada, S.; Nishimura, K. Nanometric positioning over a one-millimeter stroke using a flexure guide and electromagnetic linear motor. Int. J. Precis. Eng. Manuf. 2007, 8, 49-53.

28. Kordonskii, V.; Demchuk, S. Heat transfer in electrodynamic transducers. J. Eng. Phys. 1990, 59, 1499-1504. [CrossRef]

29. Vu, T.T.; Maeda, Y.; Aketagawa, M. Sinusoidal frequency modulation on laser diode for frequency stabilization and displacement measurement. Measurement 2016, 94, 927-933. [CrossRef]

30. Newport. DC-250 MHz Electro-Optic Phase Modulator-Models 400X. U.S. Patent 5,189,547, 26 April 2021. 